\title{
Targeted molecular therapies for ovarian cancer: An update and future perspectives (Review)
}

\author{
HIROSHI SHIGETOMI, YUMI HIGASHIURA, HIROTAKA KAJIHARA and HIROSHI KOBAYASHI \\ Department of Obstetrics and Gynecology, Nara Medical University, Nara 634-8522, Japan
}

Received February 20, 2012; Accepted May 3, 2012

DOI: $10.3892 /$ or.2012.1833

\begin{abstract}
Identification of the potential gene expression profiles of epithelial ovarian cancer and the arrival of newly targeted therapies have advanced the strategies used for treatment of this disease. This review focuses on the design of ongoing and planned clinical trials and offers a synopsis of the Englishlanguage literature for preclinical and clinical targeted therapies for epithelial ovarian cancer. Among many targeted agents, a promising, novel class of targeted drugs for special patient populations expected to improve the effectiveness of current therapy include inhibitors of angiogenesis, poly (ADP ribose) polymerase (PARP) and DNA repair mechanisms. Inhibition of PARP or homologous recombination (HR) repair mediated by Chk1 (checkpoint kinase 1) would selectively sensitize p53 mutation, BRCAness phenotype (serous type ovarian cancer) or HNF (hepatocyte nuclear factor)- $1 \beta$-overexpressing tumor cells (clear cell type ovarian cancer) to chemotherapeutic agents. The therapeutic response is likely to be limited to a targeted patient, but not to the broad population. This review discusses some of the key current developments and existing challenges.
\end{abstract}

\section{Contents}

1. Introduction

2. Study methodology

3. Antibodies

4. Small molecules

5. Conclusions

\section{Introduction}

The primary treatment for the majority of women with ovarian cancer includes a combination of surgery and chemotherapy.

Correspondence to: Dr Hiroshi Kobayashi, Department of Obstetrics and Gynecology, Nara Medical University, 840 Shijo-cho, Kashihara, Nara 634-8522, Japan

E-mail: hirokoba@naramed-u.ac.jp

Key words: targeted therapy, ovarian cancer, poly (ADP ribose) polymerase, checkpoint kinase, hepetocyte nuclear factor-1 $\beta$
Advances in surgical and chemotherapeutic management have improved progression-free survival (PFS) and overall survival (OS) over the past decade. Paclitaxel plus carboplatin combination is the primary treatment strategy in the frontline setting. Unfortunately, many women eventually relapse after adjuvant therapy, and are at great risk of recurrence. A major challenge is to understand the molecular mechanism leading to the state of resistance to standard chemotherapy.

Novel drugs are being developed to target specific molecular pathways (1). Knowledge of the pathogenesis of ovarian cancer has led to a better understanding and identification of potential molecular targets which hold great promise for personalized treatment. Overexpression of target molecules in cell lines, against murine xenografts or in human tissues has been correlated with proliferation, invasion, metastasis and prognosis. Target molecules serve as the 'master regulator', modulating the expression of hundreds of genes. Clinical trials assess the clinical utility of inhibitors/regulators of target molecules to predict progression-free and overall survival, and establish new treatment strategies that will further improve survival. It has been confirmed that the benefits of molecular targeted therapy are likely to outweigh their adverse effects, indicating the therapeutic significance of target molecules.

A growing number of new molecular targeted therapeutics are now available. These novel class of drugs are used either as single agents or in combination with chemotherapy, principally in the advanced, recurrent and metastatic settings. Agents target survival factors, angiogenesis pathways and the DNA repair machinery that are aberrantly regulated in tumors. So far, the most promising targeted agents for epithelial ovarian cancer are angiogenesis and PARP inhibitors (2). A major advancement in the treatment of cancer is the introduction of monoclonal antibodies and small molecule tyrosine kinase inhibitors (TKIs). There are several major classes of targeting compounds: antibodies that target specific growth factor receptors, or ligands for receptors, and small molecule compounds that inhibit tyrosine kinase activities or regulate the DNA repair machinery. This study reviews the most advanced molecular targets that are currently being evaluated in ovarian cancer treatment and have demonstrated efficacy as well as novel therapies.

\section{Study methodology}

The present study reviews the literature for clinical and biological studies of molecularly targeted therapies for 


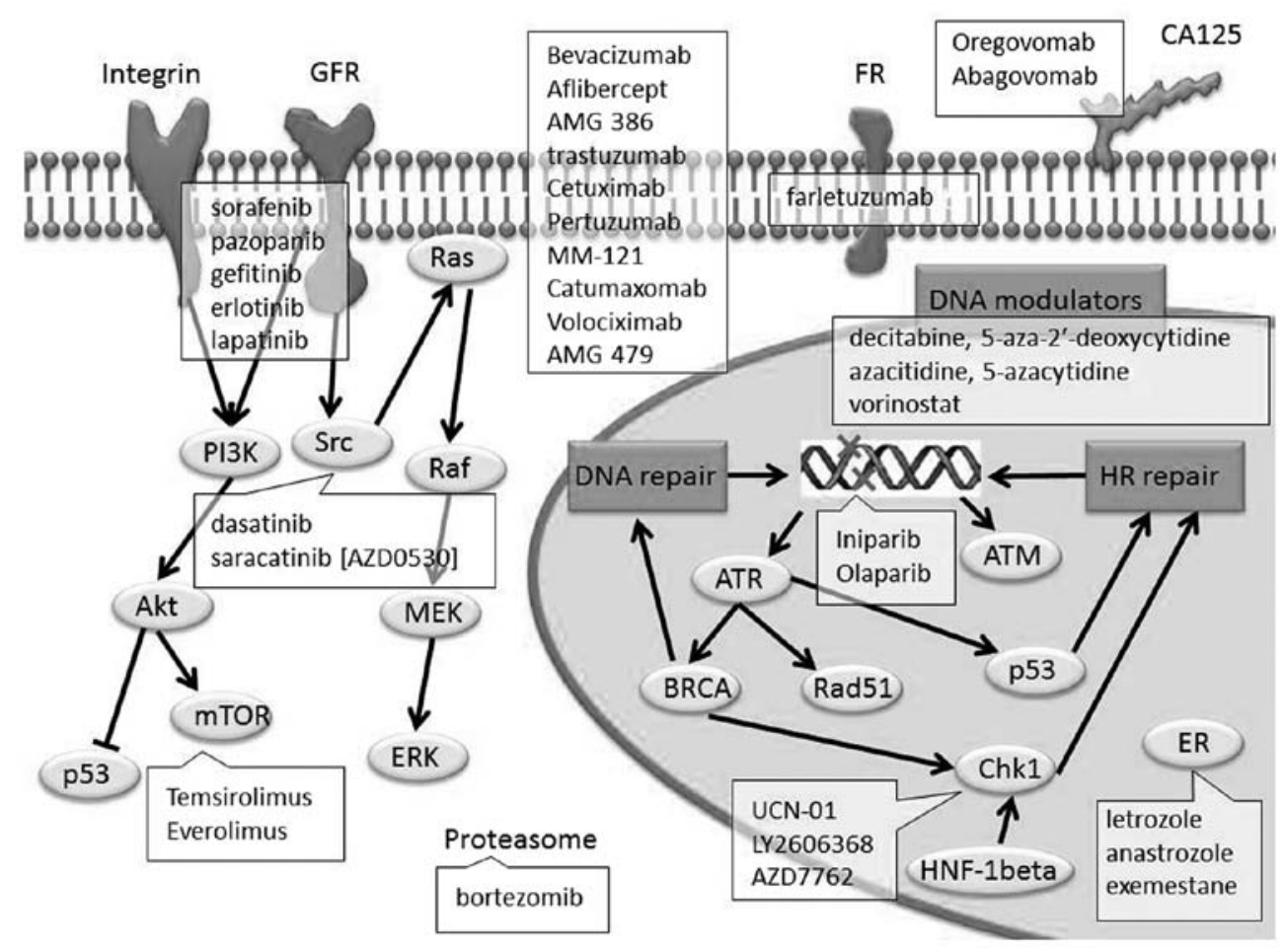

Figure 1. Targeted molecules and their signal transduction cascades in ovarian cancer. The diagram depicts the tumor signal transduction cascades and mechanism of known targeted molecule inhibitors. Targeted monoclonal antibodies for extracellular components (white box), TKIs (gray box) for each receptor signaling axis and other inhibitors (black box) are shown. The activation of adaptive compensatory mechanism may play a role in the development of resistance to targeted therapies in ovarian cancer. Strategies to prevent the compensatory pathway include intervention at different levels, i.e., at the receptor level or by blocking intracellular levels or nuclear fraction levels.

epithelial ovarian cancer. Data pertaining to fallopian tube cancer and primary peritoneal cancer were included. A computerized literature search was performed to identify relevant studies reported in the English language. All abstracts from Medline, Cochrane and the ClinicalTrials.gov electronic database were reviewed by two investigators to identify papers for full-text review. The web-based database ClinicalTrials.gov (http://clinicaltrials.gov/ct2/home), a service of the National Institutes of Health, has been constructed based on information collected from clinical trials conducted in the United States and around the world. At the time this manuscript was prepared ClinicalTrials.gov contained 822 studies for epithelial ovarian cancer. Clinical trials in this database are available for download and virtual screening. These three database were searched, combining the keywords 'targeted therapy', 'clinical trial', 'antibody', 'small molecule' or 'chemotherapy' with 'ovarian cancer'. Additionally, references in each study were searched to identify potentially missed studies. Target publications are mainly reports on human studies and animal models, as well as basic studies in gene and protein expression systems. A priori, case reports, and abstracts were not included, since abstracts do not undergo a stringent peer review process.

\section{Antibodies}

More than 100 clinical trials with monoclonal antibodies directed towards the specific antigens in patients with epithelial ovarian cancer have been reviewed and discussed $(3,4)$. Therapies with monoclonal antibodies are personalized medicines.
Targeting angiogenesis. Angiogenesis is important for tumor growth and metastasis, and might be an important target for new biological agents (1). The only clinically available anti-angiogenic agents are monoclonal antibodies and small molecule receptor TKIs (Table I and Fig. 1). The molecular mechanisms and clinical results of the promising targeted agents have been reviewed (5).

Bevacizumab. Bevacizumab is a humanized recombinant monoclonal antibody that prevents vascular endothelial growth factor (VEGF) receptor binding. Currently, more than 70 clinical trials with bevacizumab in patients with epithelial ovarian cancer or primary peritoneal cancer have been conducted to assess if bevacizumab can help control ovarian cancer. Bevacizumab monotherapy $(15 \mathrm{mg} / \mathrm{kg}$ intravenously every 21 days) in phase II clinical trials might be effective in the treatment of persistent, resistant or recurrent epithelial ovarian cancer or primary peritoneal cancer with a response rate of $16-21 \%$.

A new paradigm is emerging in which bevacizumab will be combined with conventional chemotherapeutic agents. Richardson et al reported for the first time that a retrospective chart review was conducted to investigate the response rate, PFS, safety and toxicity profile of a combination of gemcitabine, platinum, and bevacizumab for the treatment of recurrent ovarian cancer (6). The total response rate was 78\%. A complete response was achieved by $48 \%$ and $30 \%$ had a partial response. Although addition of bevacizumab to conventional chemotherapeutic agents showed remarkable activity and excellent efficacy, bevacizumab has demonstrated distinct profiles in producing 
a variety of non-hematologic toxicities, including serious toxicities, such as gastrointestinal perforation. Retrospective analysis also showed that, in heavily pretreated ovarian cancer patients, the addition of bevacizumab to metronomic oral cyclophosphamide is a safe and effective regimen with a total response rate of $44 \%$ (7). Two phase II studies of combined bevacizumab (10 mg/kg every 2 weeks) and oral cyclophosphamide $(50 \mathrm{mg} / \mathrm{day}$ ) have demonstrated total response rates of 24 and 53\%, respectively (5). The bevacizumab dose did not correlate with the therapeutic response.

The phase III clinical trials, GOG-218 and ICON7, have evaluated the efficacy and safety of bevacizumab in combination with conventional chemotherapeutic agents such as carboplatin and paclitaxel in patients with newly-diagnosed advanced ovarian cancer (8). Patients were randomly assigned to receive concurrent and maintenance bevacizumab or chemotherapy alone. The combination of bevacizumab, as compared with chemotherapy alone, significantly prolonged PFS [hazard ratio $0.75,95 \%$ confidence interval $(\mathrm{CI}) 0.68-0.83$; $\mathrm{P}<0.001]$ but showed a modest benefit, with an increase in severe gastrointestinal adverse events (1). Severe adverse events, including gastrointestinal perforation, hypertension and bleeding, were frequently observed in the clinical trials. A detailed review of ICON7 data showed that although results of these trials found no difference in OS between the two groups, the benefits with respect to OS were greater among those at high risk for disease progression $(1,8)$. To further improve efficacy, bevacizumab therapy in combination with other targeted therapy or chemotherapy was attempted, and the effects were reported (9). The combination of bevacizumab and erlotinib, an orally active EGFR TKI, failed to show any clinical benefit. The toxicity of the combination was much higher than anticipated and showed very limited additional efficacy.

Furthermore, an ongoing phase I non-randomized trial is investigating the efficacy and safety of the combination of lenalidomide $\left(\right.$ Revlimid $^{\circledR}$, thalidomide derivative, $20 \mathrm{mg}$ for 3 weeks, every 4 weeks) to pegylated liposomal doxorubicin (Doxil $^{\circledR}, 30 \mathrm{mg} / \mathrm{m}^{2}$ on Day 1) and bevacizumab $15 \mathrm{mg} / \mathrm{kg}$ on Day 1, every 3 weeks in patients with platinum resistant/ refractory ovarian cancer (NCT01202890; registered at http:// clinicaltrials.gov). Patients will be received up to 6 cycles or disease progression, followed by lenalidomide maintenance in patients with stable disease. This study is currently recruiting participants. Taken together, bevacizumab might be the most promising drug and can expand therapeutic options, but firstline bevacizumab may not be recommended.

Aflibercept. Aflibercept is a decoy receptor fusion protein combining the constant region $(\mathrm{Fc})$ of $\mathrm{IgG} 1$ with the first three domains of VEGFR1, rationally designed to block angiogenesis by targeting VEGF-A, VEGF-B and placental growth factor (10). Aflibercept was effective in preclinical models, including an in vitro and an in vivo orthotopic renal cancer model in mice. Treatment of cancer cells with aflibercept reduced tumor cell proliferation; and animals treated with aflibercept developed a reduced tumor volume by $70-80 \%$ compared with controls. Preclinical and clinical studies have been investigating the use of aflibercept alone and in combination with both chemotherapy and radiotherapy (11).

A multicenter phase II study (NCT00327444) assessed the efficacy and safety of aflibercept $(4 \mathrm{mg} / \mathrm{kg}$ every 2 weeks) in patients with advanced chemoresistant epithelial ovarian cancer and symptomatic malignant ascites (12). Aflibercept prolonged PFS [median PFS was 59.5 (95\% CI 41.0-83.0) days] and was effective in the control of refractory ascites. The adverse event profiles were similar to those for other anti-VEGF agents, including gastrointestinal disorders. The phase I/II trials were conducted to determine the pharmacokinetics, safety and efficacy of combination aflibercept and docetaxel in patients with recurrent ovarian, primary peritoneal, or fallopian tube cancer (13). The response rate was 54\% (24\% complete response and $30 \%$ partial response rate). Combining aflibercept with docetaxel resulted in greater therapeutic benefit than either agent alone with acceptable toxicity. This promising antitumor efficacy warrants further testing in a randomized trial.

$A M G$ 386. AMG 386 is a recombinant peptide-Fc fusion protein (peptibody) containing a peptide sequence that binds angiopoietin-1 and angiopoietin-2 (14). AMG 386 is an antiangiopoietin peptibody that reduces tumor angiogenesis.

A total of 6 clinical trials have been performed in order to determine the appropriate dosing, safety and clinical benefit: AMG 386 in combination with paclitaxel (NCT01204749 and NCT00479817), Doxil (NCT01281254 and NCT00770536), topotecan (NCT00770536), and paclitaxel and carboplatin (NCT01493505 and NCT01253681). The phase Ib, non-randomized NCT00770536 study of AMG $386(10 \mathrm{mg} / \mathrm{kg}$ or $15 \mathrm{mg} / \mathrm{kg}$ ) was terminated and verified on September 2011. The phase III, randomized, double-blind trial (TRINOVA-1 study and NCT01204749) of weekly paclitaxel $\left(80 \mathrm{mg} / \mathrm{m}^{2}\right.$ weekly, 3 weeks on/1 off) plus AMG 386 (weekly intravenous AMG 386, $15 \mathrm{mg} / \mathrm{kg}$ ) or placebo was also verified on September 2011. The phase III randomized, double-blind, placebocontrolled, multicenter NCT01493505 study of AMG 386 $\left(15 \mathrm{mg} / \mathrm{kg}\right.$, weekly intravenously) with paclitaxel $\left(175 \mathrm{mg} / \mathrm{m}^{2}\right)$ and carboplatin (AUC 5 or 6 ) as first-line treatment is currently recruiting participants and was verified on January 2012. The preliminary data suggest evidence of promising antitumor activity and possibly a dose-response effect (15).

Targeting human epidermal growth factor receptors (ErbB, $E G F R$ ). The ErbB (avian erythroblastic leukemia viral (v-erb-b) oncogene homolog) or EGFR (epidermal growth factor receptor) proto-oncogene family consists of four structurally-related transmembrane receptors [i.e., EGFR (ERBB1, HER1), ErbB2 (HER-2, HER2, HER-2/neu), ErbB3 (HER3), and ErbB4 (HER4)]. The downstream signaling cascades, including the RAS-RAF (v-raf murine sarcoma viral oncogene homolog)-MAP (mitogen-activated protein) kinase pathway and the PI3K (phosphatidylinositol 3-kinase)/Akt pathway, participate in the molecular pathogenesis of cancer and important tumor biologies, including cell cycle activation, proliferation, motility, adhesion, cell survival, invasion, angiogenesis and metastasis (16). Dual inhibition of both pathways has been proven to represent an attractive target for anticancer therapies in many types of cancer and may potentially exhibit favorable efficacy. HER-2 is a proto-oncogene. HER-2 gene amplification and protein overexpression have been reported in breast cancer and are associated with an aggressive clinical course and poor prognosis (17). A GOG study demonstrated, however, that HER-2 amplification has no predictive or prognostic value in ovarian cancer (18). The 


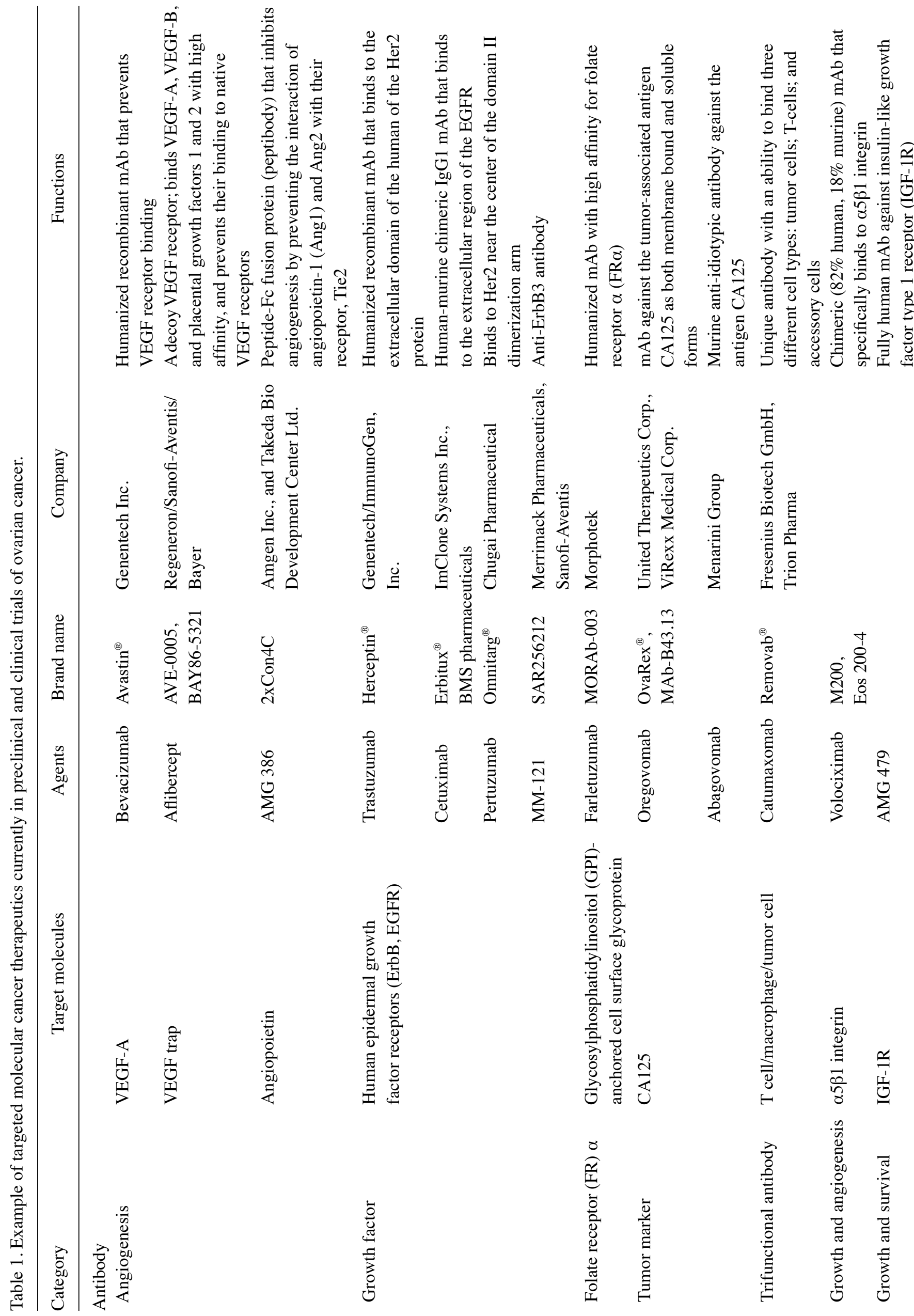




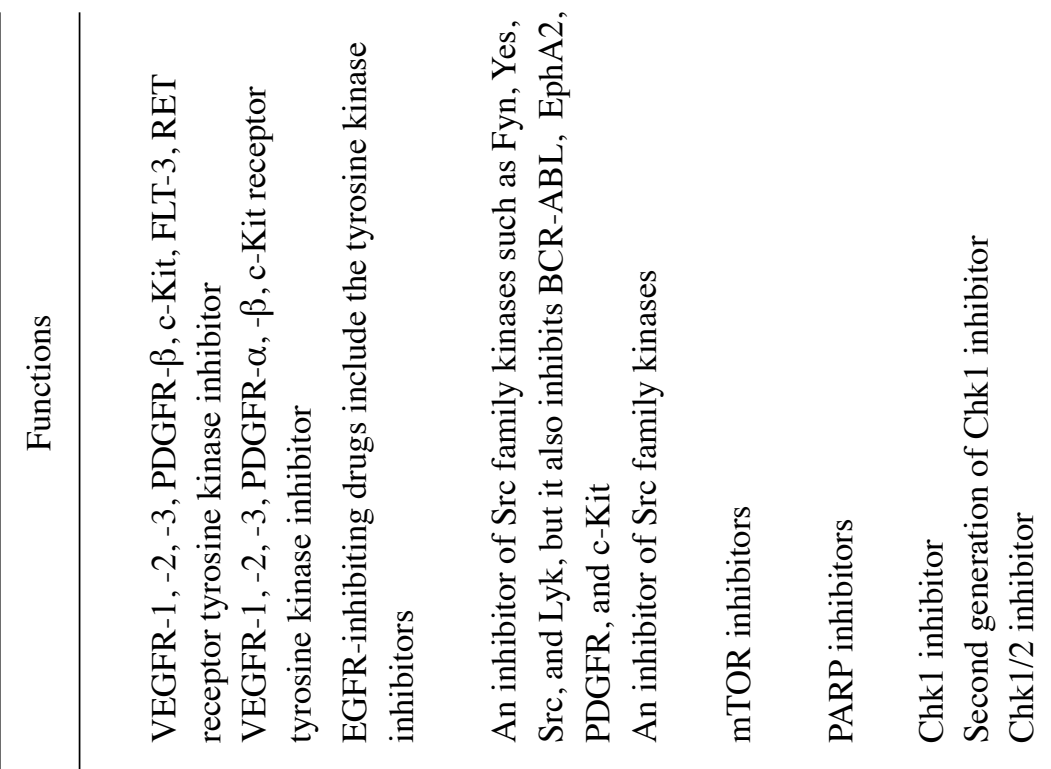

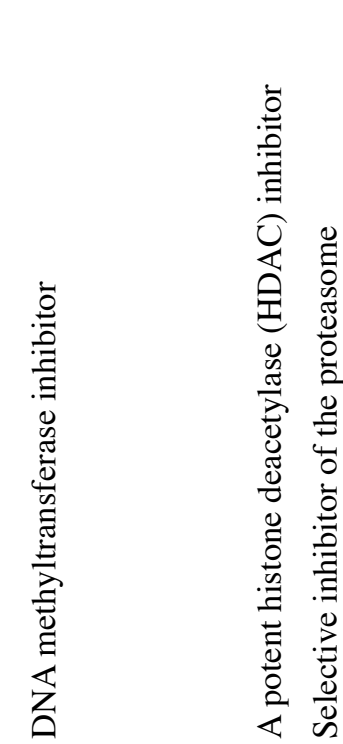

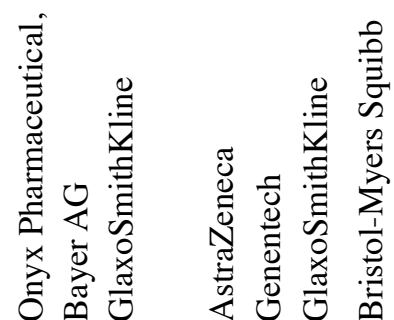

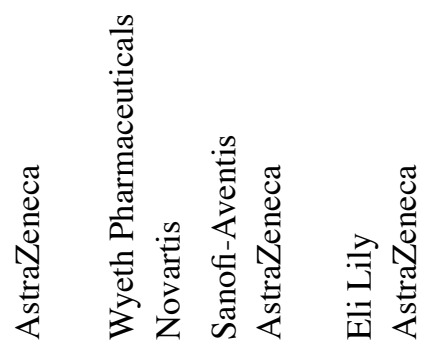

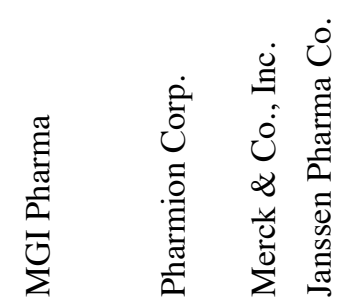

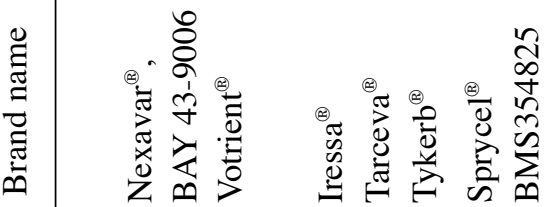

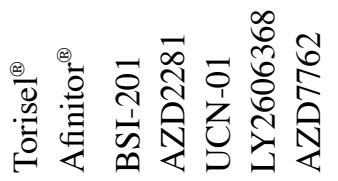

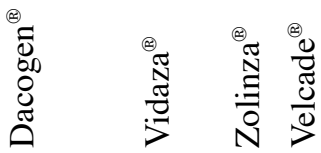

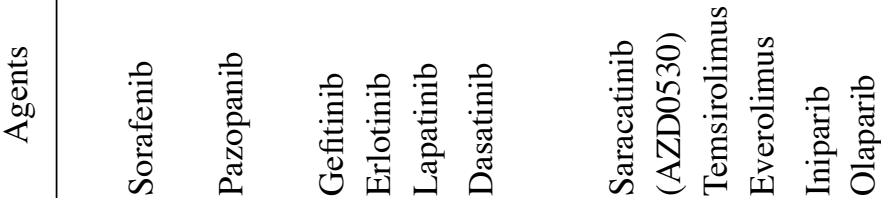

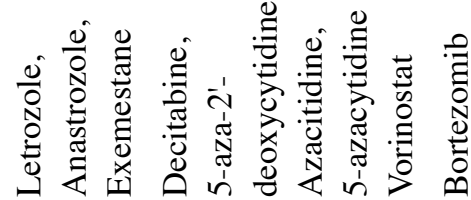

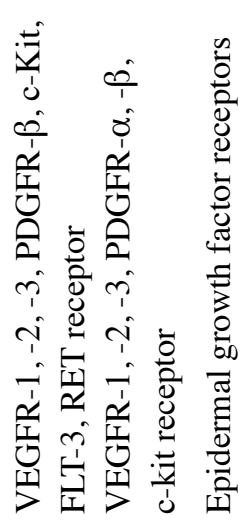

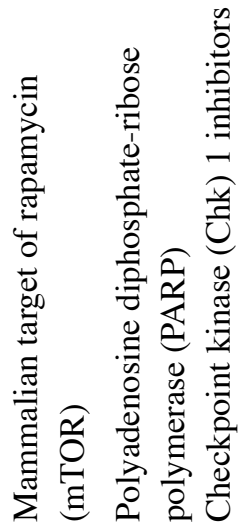

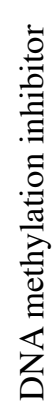

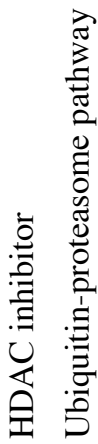


potential impact of amplification on prognosis is still controversial.

Trastuzumab. Trastuzumab (Herceptin ${ }^{\circledR}$; Genentech/ ImmunoGen, Inc.) is a humanized monoclonal antibody targeted against the human HER-2. Trastuzumab monotherapy can allow for some responses or strong activity in HER-2positive breast cancer. Favorable preclinical results in ovarian cancer have led to the development and initiation of a number of clinical trials. Ongoing and planned trials include those using trastuzumab monotherapy (NCT01195935) or trastuzumab in combination of conventional chemotherapeutic agents such as paclitaxel and carboplatin (NCT00189579) in ovarian cancer.

Cetuximab. Cetuximab (Erbitux ${ }^{\circledR}$; ImClone Systems, Inc.) is a monoclonal antibody directed against the extracellular domain of EGFR. Encouraging results from the clinical trials with cetuximab monotherapy have led to an impact on the management of patients with breast and colorectal cancer. Clinical trials have been conducted to evaluate the safety and efficacy of cetuximab monotherapy (NCT00082212) or cetuximab in combination with carboplatin (NCT00086892 and NCT00063401) in the treatment of recurrent platinum-sensitive ovarian or primary peritoneal cancer. A non-randomized phase II clinical study determined the response rate of cetuximab alone $\left(400 \mathrm{mg} / \mathrm{m}^{2}\right.$ loading dose, $250 \mathrm{mg} / \mathrm{m}^{2}$ weekly, 2 cycles), PFS, and 1-year survival with cetuximab in patients with ovarian or primary peritoneal carcinoma who have persistent or recurrent disease following several previous regimens of chemotherapy (NCT00082212). Unfortunately, results from this trial have been very disappointing, with lack of efficacy for monotherapy (19). This study has been terminated due to lack of sufficient efficacy.

Pertuzumab. Pertuzumab (Omnitarg ${ }^{\circledR}$, HER-2 dimerization inhibitor; Genentech, Inc.) is a monoclonal antibody that disrupts the interaction of HER-2 with HER-1 and HER-3, therefore blocking HER-2-driven downstream signaling (20). Pertuzumab synergistically led to increased cell death in cancer cells treated with trastuzumab.

Results from phase II trials of pertuzumab as a single agent have been disappointing (19). To date, pertuzumab has been combined with other chemotherapeutic agents to improve outcomes in three large phase II studies (21). A phase II, randomized, placebo-controlled, double-blind, multicenter clinical trial was conducted to characterize the safety and tolerability and estimate PFS of pertuzumab with gemcitabine in patients with platinum-resistant ovarian, primary peritoneal, or fallopian tube cancer (NCT00096993) (20). This study has been completed. This combination therapy is associated with better PFS (the adjusted hazard ratio was estimated to be 0.66 ). Activated HER-2 or low HER-3 mRNA expression is a predictive and valuable prognostic marker for clinical benefit from pertuzumab. Treatment selection improved the response rate (13.8 vs. $4.6 \%, \mathrm{P}<0.05)$, showing the concept of individualized or personalized targeted cancer therapy. Pertuzumab might be an exciting and promising agent that enhances the cytotoxicity of other chemotherapeutic agents (21).

Anti-ErbB3 antibody (MM-121). HER-3 is an activator of PI3K signaling in EGFR, HER-2, and hepatocyte growth factor (HGF) receptor (MET) (22). HER-2 or HER-3 heterodimerizes with the other ErbBs. The HER-2-HER-3 heterodimer is the most mitogenic. Overexpression of HER-3 has been reported in epithelial ovarian cancer. Anti-ErbB3 antibody (MM-121) abrogates phosphorylation of HER-3. A multicenter, open-label, randomized, phase II study with a combination of MM-121 plus paclitaxel vs. paclitaxel alone in patients with platinum resistant or refractory recurrent/ advanced ovarian cancers (NCT01447706) is ongoing. Targeting HER-3 with MM-121 may provide an effective strategy only for subsets of patients with upregulation of HER-3 expression. A useful biomarker must be established to select a subset of patients who stand to benefit from targeted therapy.

\section{Targeting folate receptor $(F R) \alpha$}

Farletuzumab. Folate receptor (FR)- $\alpha$ is overexpressed in ovarian cancer, which confers a tumor growth advantage. Farletuzumab is a humanized monoclonal antibody with high affinity for FR- $\alpha$ and currently under evaluation (3). Phase I-III clinical trials were conducted to characterize the safety, maximum tolerated dose, tolerability and efficacy of farletuzumab alone (NCT00428766, NCT00318370, NCT00428766 and NCT01018563) or in combination with paclitaxel (NCT00738699), carboplatin plus Doxil (NCT01004380) or carboplatin plus paclitaxel (NCT00849667) in patients with platinum-sensitive or -resistant ovarian cancer in first relapse $(4,23)$. Farletuzumab has shown antitumor activity and relatively favorable toxicity profile (23). The treatment was safe and well-tolerated in the management of heavily pretreated patients.

\section{Targeting CA125}

Oregovomab. Oregovomab (OvaRex ${ }^{\circledR}$ MAb-B43.13) is a murine monoclonal antibody specific for CA125. The CA125oregovomab complex results in immune processing and can elicit immunity against tumors, suggesting that a favorable clinical impact would be achieved using the oregovomab immunization strategy. A randomized, double-blind, placebocontrolled, phase IIb study assessed the safety and efficacy of oregovomab in patients with an elevated serum CA125 but without other evidence of disease (NCT00004064). A phase III clinical trial of intravenous oregovomab as post chemotherapy consolidation has also been conducted for epithelial carcinoma of ovarian, tubal or peritoneal origin (NCT00050375). Unexpectedly, oregovomab monotherapy failed to improve the outcomes after first-line therapy (24).

Abagovomab. Abagovomab is another murine antiidiotypic antibody against CA125. An open-label phase I trial assessed the safety and immunogenicity of abagovomab in patients with epithelial ovarian, fallopian tube, or primary peritoneal cancer (25). The phase I study confirmed the safety, tolerability and immunogenicity of abagovomab. Evaluation of clinical efficacy in the context of controlled phase III studies is warranted. An ongoing, double-blind, placebo-controlled, multicenter, phase III trial (MIMOSA) (monoclonal antibody immunotherapy for malignancies of ovary by subcutaneous abagovomab) has compared abagovomab maintenance therapy to placebo (26). This phase III trial will determine a clinical benefit for continuation maintenance chemotherapy.

Catumaxomab. Catumaxomab (Removab ${ }^{\circledR}$; Fresenius Biotech $\mathrm{GmbH}$, Trion Pharma) is a trifunctional monoclonal antibody with two different antigen-binding sites that binds 
three different cell types (27). This antibody binds to epithelial tumor cells via the adhesion molecule (EpCAM) and to $\mathrm{T}$ cells via $\mathrm{CD} 3$, and to $\mathrm{Fc} \gamma$ receptors (Fc $\gamma \mathrm{Rs}$ ) on accessory cells (natural killer cells, dendritic cells and macrophages). It was hypothesized that catumaxomab would activate $\mathrm{T}$ cells and subsequently eliminates EpCAM-positive cancer cells. The phase II randomized, open-label trial determined the efficacy of intraperitoneal catumaxomab therapy in patients with platinum-resistant or -refractory epithelial ovarian cancer (EudraCT 2004-000723-15) (28). Catumaxomab was well-tolerated but had modest activity. The phase II/III randomized study was also conducted in EpCAM positive cancer patients with symptomatic malignant ascites (including ovarian cancer) using catumaxomab (NCT00836654). This study has been completed and was being analyzed at the time this manuscript was under preparation. Intraperitoneal infusion of catumaxomab is also in an ongoing phase III study (NCT00822809) as a treatment of malignant ascites due to epithelial cancer (4).

Volociximab. Volociximab, a first-in-class chimeric monoclonal antibody against ( $\alpha 5 \beta 1)$ integrin, blocks the interaction between $\alpha 5 \beta 1$ and extracellular matrix ligands, such as fibronectin, vitronectin, laminin, collagens and other plasma membrane proteins. Since the $\alpha 5 \beta 1$ signaling pathway is downstream of growth factor stimulation, volociximab is considered to be a potent inhibitor of proliferation and angiogenesis. The phase II, multicenter, single-arm, two-stage study evaluated the efficacy, safety, and tolerability of weekly singleagent volociximab in platinum-resistant, advanced epithelial ovarian or primary peritoneal cancer (NCT00635193) (29). Volociximab has been well tolerated as weekly monotherapy, but there is insufficient evidence to reach conclusions about the effectiveness of volociximab.

\section{Targeting IGF- $1 R$}

$A M G 479$. AMG 479 is a human monoclonal antibody against the human insulin-like growth factor type 1 receptor (IGF-1R). This antibody inhibits cancer cell proliferation via disruption of the IGF-1R-dependent PI3K/Akt and MAP kinase pathways. The biological effects of AMG 479 were examined as a monotherapy and in combination with a chemotherapeutic agent. Two clinical trials have been performed in order to determine the appropriate dosing, safety and clinical benefit: AMG 479 to paclitaxel and carboplatin first line chemotherapy in patients with optimally debulked $(<1 \mathrm{~cm})$ FIGO stage III and IV (positive pleural cytology only) ovarian epithelial (including fallopian tube and primary peritoneal) carcinoma (NCT00718523) and AMG 479 alone in patients with recurrent platinum-sensitive ovarian epithelial (including fallopian tube and primary peritoneal) carcinoma failing frontline chemotherapy (NCT00719212). These studies have not been completed. The NCT00718523 and NCT00719212 studies are currently recruiting participants as verified on December 2011 and August 2012, respectively.

\section{Small molecules}

\section{Targeting VEGFR}

Sorafenib. Sorafenib (Nexavar ${ }^{\circledR}$, BAY 43-9006) is an orally available multi-targeted TKI that blocks the activation of
RAF (v-raf-1 murine leukemia viral oncogene homolog), c-Kit (v-Kit Hardy-Zuckerman 4 feline sarcoma viral oncogene ho), FLT-3 (fms-related tyrosine kinase 3), RET (ret proto-oncogene), VEGFR-2, VEGFR-3 and PDGFR (platelet-derived growth factor receptor) $-\beta$. Sorafenib improved PFS in both first-line and second-line settings in patients with advanced renal cell cancer. Despite advances in sorafenib therapy, these treatments rarely yield complete responses and thus are not curative.

In epithelial ovarian cancer, ongoing and planned clinical trials include those using sorafenib monotherapy (NCT00522301, NCT00791778 and NCT00093626) or sorafenib in combination of conventional chemotherapeutic agents such as topotecan (NCT00526799 and NCT01047891), gemcitabine (NCT00096395), and paclitaxel and carboplatin (NCT00390611 and NCT00096200). A open-label, multiinstitutional, phase II study investigated the efficacy, safety and tolerability of sorafenib monotherapy in patients with recurrent epithelial ovarian cancer or primary peritoneal cancer (NCT00093626) (30). Patients (3.4\%) achieved partial responses; $34 \%$ demonstrated stable disease. Single agent sorafenib therapy confers modest activity with substantial toxicity. Another phase II study assessed the efficacy, safety and tolerance of sorafenib monotherapy as a third line therapy in patients with epithelial ovarian cancer or primary peritoneal cancer (31). Since the trial results have been disappointing as third-line treatment for ovarian cancer, this trial was terminated.

Subsequently, the safety and efficacy of the combination of sorafenib and conventional chemotherapeutic agents has been evaluated in several phase I/II clinical trials. The NCT00526799 clinical study determined the efficacy and safety of sorafenib (400 mg twice daily on days 1-28 of each 4-week cycle) and weekly topotecan $\left(3.5 \mathrm{mg} / \mathrm{m}^{2}\right.$ intravenously, weekly) in patients with platinum-resistant/refractory ovarian cancer or primary peritoneal cancer (32). Patients (16.7\%) achieved partial responses; $46.7 \%$ demonstrated stable disease. Grade 3/4 toxicities remain significant: leukocytopenia/neutropenia, thrombocytopenia, anemia, fatigue, nausea, vomiting, and hand-foot syndrome. The combination of topotecan with sorafenib is likely to yield only modest efficacy alone. The Princess Margaret Hospital Canada Phase II Consortium study was designed to assess the treatment efficacy and safety profile of sorafenib ( $400 \mathrm{mg}$ twice daily) and gemcitabine (1000 $\mathrm{mg} / \mathrm{m}^{2}$ intravenous) combination for patients with recurrent epithelial ovarian cancer (33). Patients (4.7\%) had a partial response; and $23.3 \%$ maintained objective response or stable disease lasting longer than 6 months. CA125 marker response was $27.9 \%$. The median PFS and OS were 5.4 and 13.0 months, respectively.

Treatment-related hematologic toxicity suggests caution to management. Non-hematologic adverse events include handfoot syndrome, fatigue, hypokalemia, and diarrhea. Because of no significant evidence of increased efficacy, the combination of sorafenib and gemcitabine does not warrant further evaluation. The phase II trial assessed the activity and tolerability of the combination therapy of carboplatin (AUC 5)/paclitaxel $\left(175 \mathrm{mg} / \mathrm{m}^{2}\right)$ with sorafenib (400 mg twice daily) in patients with primary advanced epithelial ovarian cancer (34). All patients had to stop the protocol therapy because of severe 
toxicities. The addition of sorafenib to carboplatin/paclitaxel chemotherapy was not a feasible therapeutic approach.

\section{Targeting VEGFR, PDGFR and c-Kit}

Pazopanib. Pazopanib is an orally bioavailable, multitargeted TKI that inhibits angiogenesis targeting VEGFR1-3, PDGFR $\alpha / \beta$, FGFR (fibroblast growth factor receptor) 1,3 and 4, KIT and RET (35).

Ongoing clinical trials include those using pazopanib monotherapy (NCT01262014, NCT00281632, NCT00866697 and NCT01227928) or pazopanib in combination of conventional chemotherapeutic agents such as cyclophosphamide (NCT01238770), Doxil (NCT01035658), paclitaxel (NCT01468909), cisplatin (NCT01165385), and paclitaxel and carboplatin (NCT01402271 and NCT00561795) in ovarian cancer. The multicenter phase I/II clinical trial was designed to evaluate the optimal dose, safety and efficacy for pazopanib $(400,600$ and $800 \mathrm{mg} /$ day) in combination with oral cyclophosphamide $(50 \mathrm{mg} /$ day $)$ as salvage treatment of patients with recurrent, platinum-resistant, pre-treated ovarian cancer (35). Friedlander et al (36) reported that $31 \%$ of patients had a CA125 response to pazopanib, suggesting that pazopanib has activity in terms of CA125 response in recurrent ovarian cancer. Pazopanib has proven, albeit modest, promising clinical benefit as monotherapy in phase II studies in ovarian cancer. Diverse anti-angiogenic drugs are under investigation.

\section{Targeting PDGFR and c-Kit}

Imatinib.Imatinib mesylate (Gleevec ${ }^{\circledR}$; Novartis Pharmaceuticals Corp.), a small-molecule TKI, selectively inhibits ABL (c-abl oncogene 1) kinase, chimeric BCR-ABL fusion oncoprotein of chronic myeloid leukemia (CML), transmembrane receptor KIT and PDGFRs (37). It is approved for the treatment of CML and gastrointestinal stromal tumor (GIST) (38).

The safety and efficacy of imatinib have been currently evaluated in several clinical trials in patients with advanced, persistent or recurrent ovarian cancer. Ongoing clinical trials have investigated the safety and efficacy of imatinib monotherapy (NCT00510653, NCT00039585, NCT00042952, NCT00036751 and NCT00041041) or imatinib in combination with conventional chemotherapeutic agents such as docetaxel (NCT00216112), gemcitabine (NCT00928642) and paclitaxel (NCT00840450) (37). Some preliminary data showed that the effect of the advances on clinical outcomes has been disappointing; few patients had sustained responses or stable disease; and treatment with imatinib did not prolong the PFS (39).

Targeting epidermal growth factor receptors (ErbB, EGFR) Gefitinib, erlotinib and lapatinib. The representative TKIs inhibiting EGFR kinase include gefitinib (Iressa ${ }^{\circledR}$; AstraZeneca Pharmaceuticals), erlotinib (Tarceva $\left.{ }^{\circledR}\right)$ and lapatinib (Tykerb ${ }^{\circledR}$; GlaxoSmithKline), which targets both EGFR and HER-2. They are currently undergoing clinical development. Clinical trials have evaluated the efficacy and safety of lapatinib monotherapy (NCT00113373) or lapatinib in combination of conventional chemotherapeutic agents such as carboplatin (NCT00317434), topotecan (NCT00436644, NCT00888810), paclitaxel (NCT00313599) and carboplatin/ paclitaxel (NCT00316407) in ovarian cancer. Blank et al have investigated whether erlotinib (150 mg daily) in combination with carboplatin/paclitaxel improved pathological complete response (pCR) in patients with stage III-IV epithelial ovarian, fallopian tube, or primary peritoneal cancers (40). Utilizing erlotinib in combination with standard therapy did not improve pCR rates. The phase II trial (NCT00130520) assessed the efficacy and safety of the combination of erlotinib and bevacizumab in patients with recurrent ovarian, primary peritoneal or fallopian tube cancer. No combination regimen has proven superior to bevacizumab monotherapy as a second-line treatment. Patients treated with erlotinib and bevacizumab are at high risk for severe and fatal gastrointestinal perforation. The study was therefore terminated due to the disappointing results of targeted therapy and severe toxicity in this setting (41).

A phase II trial evaluated the activity and tolerability of lapatinib $(1,500 \mathrm{mg} / \mathrm{day})$ in patients with recurrent or persistent epithelial ovarian cancer or primary peritoneal carcinoma, measurable disease, and up to 2 prior chemotherapy regimens for recurrent disease (42). Lapatinib has minimal activity. Furthermore, the regimen of lapatinib (750 mg daily) in combination with carboplatin in patients with platinumsensitive recurrent ovarian cancer was associated with poor treatment response and severe toxicity (43). Weroha et al (44) have designed a phase II clinical trial to evaluate the efficacy and toxicity profile of concomitant topotecan and lapatinib in patients with platinum-refractory or resistant epithelial ovarian/peritoneal cancer. The study was terminated because of insufficient efficacy and severe adverse effects such as severe neutropenia, thrombocytopenia, and diarrhea. The HER-2 and EGFR targeted therapies initially regarded as promising have been abandoned due to the lack of clinical efficacy. Despite the high rates of EGFR protein overexpression in ovarian cancer cells, response rates (0-5.9\%) from phase II trials of erlotinib, gefitinib and lapatinib are very poor (45). EGFR targeted treatment to date has no effect when given as monotherapy and as an adjunct to chemotherapy.

\section{Targeting Src}

Dasatinib and saracatinib (AZD0530). Dasatinib (Sprycel ${ }^{\circledR}$, BMS-354825) is an oral, multitargeted inhibitor of receptor tyrosine kinases, including SFKs (Src family kinases), such as Src (v-src sarcoma viral oncogene homolog), Lck (lymphocyte-specific protein tyrosine kinase), and Yes (v-yes-1 Yamaguchi sarcoma viral oncogene homolog), Bcr-Abl fusion protein, c-Kit, PDGFR, FAK (focal adhesion kinase) and EphA2 (EPH receptor A2) (46). In vivo studies showed that dasatinib inhibits ovarian cancer cell growth, invasion and metastasis as well as induces autophagy and apoptosis (47). Preclinical studies demonstrated that dasatinib-induced inhibition of receptor tyrosine kinase signalling is a potential target for the therapeutic intervention in several solid tumors, including ovarian cancer (46). Dasatinib has synergistic activity with paclitaxel and carboplatin in ovarian cancer cells. Ongoing clinical trials of dasatinib in combination with paclitaxel and carboplatin were conducted to assess the efficacy and safety in patients with advanced, persistent or recurrent epithelial ovarian, fallopian tube, or primary peritoneal cancer. These studies will further investigate the potential anticancer benefits of 
dasatinib across all stages of ovarian cancer. In addition, clinical trials have currently evaluated the efficacy and safety of dasatinib/saracatinib monotherapy (NCT00671788) or dasatinib/saracatinib in combination of conventional chemotherapeutic agents such as paclitaxel (NCT01196741) and carboplatin/paclitaxel (NCT00672295 and NCT00671788) in ovarian cancer.

Targeting mammalian target of rapamycin (mTOR) signaling pathway

Temsirolimus and everolimus. Growth factor receptors containing tyrosine kinases act upstream of the PI3K/Akt/ mTOR pathway, which plays an important role in tumor progression and drug resistance. Sequential phase II studies were conducted to evaluate the safety and efficacy of singleagent activity of temsirolimus in women with recurrent or metastatic chemotherapy-naive or chemotherapy-treated endometrial cancer (48). Temsirolimus achieved a sufficient objective response rate as treatment for endometrial cancer.

Based on promising preclinical data, mTOR inhibitors are currently being evaluated in several phase I/II trials in patients with ovarian cancer. Clinical trials have been currently conducted to evaluate the efficacy and safety of mTOR inhibitor monotherapy [temsirolimus (NCT00926107, NCT01460979 and NCT00429793), everolimus (NCT01149434)] or mTOR inhibitors in combination with conventional chemotherapeutic agents such as Doxil (NCT00982631), vinorelbine (NCT01155258), topotecan (NCT00523432), carboplatin/Doxil (NCT01281514), paclitaxel/carboplatin (NCT01196429 and NCT00408655), cediranib (VEGFR/PDGFR inhibitor and NCT01065662) and bevacizumab (NCT01031381, NCT00886691 and NCT01010126) in ovarian cancer. A phase II study determined the efficacy of temsirolimus monotherapy in persistent and recurrent epithelial ovarian and primary peritoneal cancers (49). Despite extensive clinical development efforts, temsirolimus has modest activity. A phase I study assessed the toxicities of weekly temsirolimus and topotecan in the treatment of advanced or recurrent gynecologic malignancies (50). Dose-limiting toxicity was myelosuppression and limited treatment continuation. Another phase I study assessed the safety, tolerability, recommended phase II dose, and antitumor activity of the combination of carboplatinpaclitaxel-temsirolimus (51). The combination regimen was tolerated and thought to be effective. Although clinical activity was low compared with the benefit expected, this approach warrants further investigation on the clinical utility of mTOR inhibitors. Recent clinical study showed that patients with PIK3CA (phosphoinositide-3-kinase, catalytic, $\alpha$ polypeptide) mutations treated with PI3K/Akt/ mTOR inhibitors demonstrated a higher response rate than patients without mutations (52). Further investigations will be necessary to clarify the efficacy of mTOR inhibitors for this subgroup.

\section{Targeting DNA repair machinery}

BRCAness in epithelial ovarian cancer. The breast cancer susceptibility gene BRCA pathway is committed to the repair of DNA interstrand cross-links. Cancer cells with BRCA1 or BRCA2 germline mutations are defective in DNA repair by homologous recombination (HR). Impaired ability to repair DNA increases their chemosensitivity and shows a better survival compared to the sporadic non-mutation group (53).

Dysfunction in the BRCA1 or BRCA2 genes/proteins might be due to either inherited or somatic mutations, due to epigenetic inactivation or dysfunction of upstream targets of the BRCA pathway. In fact, cancers arising in a background of defective BRCA function (BRCAness) are more sensitive than other cancers to DNA-damaging agents. The BRCAness plays an important role in selection of cytotoxics or molecular targeted agents. Ovarian cancer patients with a BRCAness may benefit from tailored treatments (54).

Polyadenosine diphosphate-ribose polymerase inhibitors. Poly (ADP-ribose) polymerase (PARP) catalyzes the addition of ADP-ribose units to DNA, histones and DNA repair enzymes. PARP is critical for single-strand DNA breaks via the base excision repair pathway (55). In the presence of defective HR repair, single-strand DNA breaks lead to doublestrand DNA breaks. Both hereditary ovarian cancer with BRCA mutations and sporadic ovarian cancer with BRCAness not expressing functional BRCA proteins are characterized by defects in HR DNA repair. Tumors with BRCA mutations or BRCAness are sensitive to DNA damaging agents $(53,55)$. Inhibition of PARP accumulates unrepaired or unresolved single-strand DNA breaks. PARP inhibitors might be effective in tumors with BRCA mutations or BRCAness $(56,57)$. In this scenario, PARP inhibition leads to synthetically lethality.

Several PARP inhibitors, including olaparib (AstraZeneca), iniparib (BSI-201, BiPar Sciences Inc./Sanofi-Aventis), veliparib (Abbott Laboratories), AG014699 (PF-01367338, Pfizer Inc.), MK-4827 (Merck \& Co., Inc.) and CEP-9722 (Cephalon Inc.), are being evaluated in the preclinical and clinical setting (58). The PARP inhibitors remain a major focus of ongoing research. Clinical studies of PARP inhibitor alone or in combination with cytotoxic agents were conducted to assess the safety and toxicity in patients with breast or ovarian cancers having BRCA1 or BRCA2 mutations.

Iniparib. Iniparib (4-iodo,3-nitrobenzamide) prevents PARP-1 activation by DNA breaks through targeting the zinc finger domain. Iniparib induces $\gamma-\mathrm{H} 2 \mathrm{AX}$ (histone $\mathrm{H} 2 \mathrm{~A}$, a marker of DNA damage) expression and causes $\mathrm{G} 2 / \mathrm{M}$ cell cycle arrest. The results of a follow-up phase III study of iniparib therapy in triple-negative breast cancer failed to show any clinical benefit, as it did not meet the prespecified criteria for PFS and OS (55). Ongoing, multicenter, single-arm clinical studies have been conducted to assess the efficacy and safety of carboplatin/gemcitabine in combination with iniparib in patients with platinum-sensitive (NCT01033123) or platinumresistant (NCT01033292) recurrent ovarian cancer.

Olaparib. Olaparib (AZD2281, $400 \mathrm{mg}$ twice a day) is a small-molecule, potent oral PARP inhibitor. Clinical trials have evaluated the efficacy and safety of oral olaparib monotherapy (NCT00679783, NCT00494442, NCT00753545, NCT00516373 and NCT01078662) or olaparib in combination of conventional chemotherapeutic agents, such as carboplatin (NCT01237067, NCT00647062 and NCT01445418), carboplatin/paclitaxel (NCT01081951 and NCT00516724), and Doxil (NCT00628251) in ovarian cancer.

As expected, olaparib has antitumor activity in ovarian cancer patients with BRCA1/2 mutations (59). A phase II, 
multicenter, open-label, non-randomized study assessed the safety and tolerability of olaparib (400 mg twice a day) in patients with or without BRCA1 or BRCA2 mutations with advanced triple-negative breast cancer or high-grade serous and/or undifferentiated ovarian cancer (60). Patients (41\%) with BRCA mutations achieved objective response and responses were also seen in $24 \%$ of those without mutations. This study suggests that olaparib represents a promising treatment strategy for women with ovarian cancer with BRCA mutations. The phase I non-randomized, open label trial has investigated the efficacy of the combination of olaparib to carboplatin in BRCA1/2 familial ovarian cancer and sporadic triple negative ovarian cancer (NCT01445418). A phase Ib dose escalation study of MK-4827, a novel PARP inhibitor, in combination with Doxil has been conducted in patients with platinum resistant/refractory high grade serous ovarian cancer (NCT01227941). These candidate agents warrant further clinical outcome and analysis.

\section{Targeting checkpoint kinase (Chk) 1}

LY2606368 and AZD7762. Exposure to chemotherapeutic agents leads to cell cycle arrest and subsequent protection of tumor cells from death. The Chk1 (checkpoint kinase 1) is a key regulator in this response. Chk1 is a serine/threonine kinase and arrests cell cycle progression from G2 phase to mitosis in response to DNA damage. Chk1 plays a key role in resistance to chemotherapeutic agents. Therefore, the chemosensitivity can be enhanced with treatment with a Chk1 inhibitor. Inhibitors of Chk1, UCN-01 (7-hydroxystaurosporine), LY2606368 (Eli Lilly), AZD7762 \{(5-(3-fluorophenyl)-3-ureidothiophene- $\quad \mathrm{N}-[(\mathrm{S})$ piperidin-3-yl]-2-carboxamide; AstraZeneca\}, and CHIR-124 \{(S)-3-(1H-benzo[d]imidazol-2-yl)-6-chloro-4-(quinuclidin-3ylamino)quinolin-2(1H)-one\}, have a potential antineoplastic activity. CHIR-124 specifically potentiates the cytotoxicity of topoisomerase I activity. Clinical trials evaluated the efficacy and safety of UCN-01 in combination of conventional chemotherapeutic agents such as topotecan (NCT00072267 and NCT00045175) in ovarian cancer. Currently, antitumor potential of the combination of UCN-01 and topotecan has been disappointing (61). Another clinical trial has been planned to evaluate the efficacy and safety of LY2606368 monotherapy (NCT01115790) in advanced ovarian cancer. Three clinical trials have also been conducted to evaluate the efficacy and safety of AZD7762 alone and in combination of conventional chemotherapeutic agents such as gemcitabine (NCT00413686 and NCT00937664) and irinotecan (NCT00473616) in patients with advanced solid malignancies. The preliminary data suggest that Chk1 inhibitors potentiate the cytotoxicity of DNA-damaging agents and reverse tumor cell resistance to chemotherapeutic agents.

Both cell cycle checkpoint abrogation and HR repair inhibition contribute to sensitization by Chk1 inhibition (62). A growing body of evidence supports that inhibition of Chk1 selectively abrogates the repair of damaged DNA, sensitizes cancer cells to radiotherapy, or increases cancer cell death in the presence of p53 mutations (62). Chk1 inhibitors can lead to an accumulation of damaged DNA and may promote genomic instability and apoptosis. Furthermore, HR is a complex process involving many components including ATM (ataxia telangiectasia mutated), ATR (ataxia telangiectasia and Rad3 related), Chk1, RAD51 and its homologues. Tumor cells become more sensitive to radiation after treatment with the inhibitor (KU-60019) of ATM, an upstream target of Chks. Loss of function in any of these components may confer sensitivity to PARP inhibitors.

Function of Chkl inhibitors in clear cell carcinoma of the ovary. Conceptually, we attempt to explain the therapeutic efficacy of Chk inhibitors in clear cell carcinoma (CCC) of the ovary and speculate how the treatment with these agents might be beneficial. CCC is an aggressive ovarian malignancy with a poor prognosis.

Recent studies based on genome-wide expression analysis technology have noted specific expression of a transcription factor hepatocyte nuclear factor-1 $\beta$ (HNF-1 $\beta$ ) in CCC (63). Analysis of the collected proteomic data revealed that a majority of the identified proteins relate to each other in a functional network centered on HNF-1 $\beta$. The integration of this study successfully identifies a set of candidate susceptibility genes for HNF-1 $\beta$. The preliminary data showed that Chk1 protein is persistently activated in the HNF-1 $\beta$-overexpressing CCC cells (64). Clinically, relevant drugs targeting proteins connected in the HNF-1 $\beta$ network may be the Chk1 inhibitors. Compared to HNF-1 $\beta$-negative cells, HNF-1 $\beta$-overexpressing cells exhibit resistance to a chemotherapeutic cytotoxic effect. Chk1 might function in the cellular survival pathways that enhance DNA damage repair, thereby granting chemoresistance. Chk1 inhibition selectively chemosensitizes HNF-1 $\beta$-overexpressing CCC cells in vitro (64). The Chk1 inhibitor might be a novel target for developing cancer therapeutics in the HNF-1 $\beta$-positive cells that can potentially enhance the sensitivity to chemotherapeutic agents acting via induction of DNA damage and apoptosis. The efficacy of the combination and sequence of Chk1 inhibitor and conventional chemotherapeutic agent administration is cell dependent and suggests that responses vary based on tumor specific characteristics, HNF-1 $\beta$ positive or -negative. Based on these findings, inhibition of HR repair by Chk1 inhibitor may be a useful strategy for selectively inducing a BRCAness-like phenotype in HNF-1 $\beta$-positive CCC cells.

\section{Other molecules}

Targeting estrogen receptor. Estrogen receptor (ER) is an important regulator in ovarian carcinogenesis and promotion. The third-generation aromatase inhibitors, letrozole, anastrozole and exemestane, are now in clinical use in breast cancer (38). Clinical studies have been conducted to evaluate the efficacy and safety of letrozole (NCT00505661 and NCT00634894), anastrozole, and exemestane (NCT00261027) alone or in combination of targeted therapeutic drug gefitinib (NCT00181688) in ovarian cancer. A phase II clinical study assessed the efficacy of letrozole $2.5 \mathrm{mg}$ daily in previously treated ER-positive ovarian cancer patients (65). Partial response was achieved by $9 \%, 42 \%$ had stable disease and $26 \%$ had a PFS of $>6$ months. A subgroup of patients, for example endometrioid ovarian carcinoma, may benefit from aromatase inhibitors. Aromatase inhibitors may show a significant level of activity and are currently under investigation in ovarian cancer. 
Targeting DNA methylation (decitabine and azacitidine). DNA methylation profiling studies highlight the relevance of the epigenetic component and verification of clinical utility. DNA methylation is catalyzed by the DNA methyltransferases (DNMTs). Evidence has accumulated that aberrant DNA methylation is frequent in the malignancies and plays a key role in epigenetic silencing of transcription, leading to regulation of the chromatin status. DNA methylation and inactivation of tumor suppressor genes contribute to genomic instability and lead to carcinogenesis. Therapeutics directed towards epigenetic mechanisms include methylation inhibitors and histone deacetylase (HDAC) inhibitors.

DNMT inhibitors, decitabine and azacitidine, have been recognized as promising candidate anticancer drugs: they have demonstrated clinical efficacy in hematologic malignancies (66). Based on the previous findings, phase I/II trials assessed the safety and efficacy of decitabine as a sensitizer to carboplatin in platinum-resistant recurrent ovarian cancer (NCT00477386 and NCT00748527). Results from this study showed a meaningful response: patients with complete response or stable disease for $>6$ months were observed, which may warrant further clinical trials. Another hypomethylating agent, azacitidine, has been shown to induce responses in hematologic malignancies. A phase I study assessed the safety of azacitidine in combination of carboplatin and paclitaxel (NCT00842582) in newly diagnosed ovarian cancer. Phase I/ II clinical trials of the sequential combination of azacitidine and carboplatin have also been conducted in patients with platinum-resistant or platinum-refractory epithelial ovarian cancer. Azacitidine-carboplatin sequential treatment can reverse platinum resistance, and merits further evaluation.

Targeting histone deacetylase (vorinostat). Histone deacetylases (HDACs) remove acetyl groups which lead to a more compact chromatin conformation and gene repression. Modulating acetylation with HDAC inhibitors has anticancer effects such as death, apoptosis, and cell cycle arrest in preclinical and clinical models. One of these compounds is vorinostat (suberoylanilide hydroxamic acid, SAHA). A phase II trial assessed the efficacy and safety of vorinostat alone in patients with recurrent or persistent epithelial ovarian or primary peritoneal carcinoma (67). Vorinostat had minimal activity as a single agent. The ongoing phase I/II single-center trial has investigated the activity and toxicities of the combination of oral vorinostat to paclitaxel plus carboplatin in treating patients with advanced recurrent, platinum-sensitive ovarian cancer (NCT00772798). Combination of vorinostat, carboplatin and gemcitabine plus vorinostat maintenance were also conducted in patients with recurrent, platinum-sensitive epithelial ovarian, fallopian tube, or peritoneal cancer (NCT00910000). These clinical trials are still ongoing.

Targeting the ubiquitin-proteasome pathway (bortezomib). The ubiquitin proteasome pathway represents the major system for intracellular protein degradation. Altered ubiquitin proteasome pathway is frequently encountered in many malignancies. The proteasome inhibitor bortezomib (Velcade ${ }^{\circledR}$ ) proved to be active against B-cell lineage malignancies such as multiple myeloma. Bortezomib has been under clinical investigation for a number of cancers, but limited preclinical studies with this agent have been conducted in ovarian cancer.
Clinical trials have been conducted to evaluate the efficacy and safety of bortezomib monotherapy (NCT00023712) or bortezomib in combination of conventional chemotherapeutic agents such as carboplatin (NCT00059618, NCT00028912 and NCT01074411), Doxil (NCT00610792 and NCT00237627), gemcitabine (NCT00620295) and paclitaxel (NCT00667641) in ovarian cancer. Clinical trials with bortezomib therapy are ongoing for the treatment of advanced or recurrent ovarian cancers.

\section{Conclusions}

The current standard treatment for epithelial ovarian cancer is multimodal, involving cytoreductive surgery and empirical combination chemotherapy, paclitaxel and carboplatin given every 3 weeks. Advances in the delivery of chemotherapy using the intraperitoneal route or the dose-dense strategy have been made in the treatment of this disease and have improved survival after initial therapy. However, most of advanced ovarian cancer patients will ultimately develop recurrence. Second- or above-line chemotherapy is palliative. The availability of new agents will further improve prognosis for the advancement or recurrent ovarian cancer.

The new technologies of proteomics have the capacity to advance our understanding of new directions for the treatment of this disease. A new generation of molecularly targeted cancer drugs used in combination with chemotherapy will have greater efficacy relative to traditional cytotoxic chemotherapeutic agents alone. Their goal are to minimize toxicity. This review discusses strategies and outcomes of the current regimens of molecular targeted therapy for advanced or recurrent ovarian cancer, and also aims at highlighting the challenges associated with the integration of novel therapies that hold great promise in recent preclinical studies into clinical practice.

Targeted therapies remain an active area of investigation and have altered the management of many hematologic malignancies and solid tumors (5). Diverse molecularly targeted drugs are under investigation, and direct targeting of this pathway can be achieved by sequestration of target protein using monoclonal antibodies or engineered binding site molecules, blockade of the growth factor receptors with monoclonal antibodies or inhibition of receptor associated tyrosine kinase with low molecular weight inhibitors, or modulation of target proteins associated with the DNA repair pathway and chromatin remodeling in the nuclear fractions. The clinical success of imatinib in the treatment of chronic myelogenous leukemia made a paradigm shift: the fusion Bcl-Abl tyrosine kinase has a significant impact on treatment response. Beyond imatinib, ongoing and planned clinical studies are exploring monotherapy or a wide range of combination strategies in many solid tumors. Currently, there are many preclinical studies that apply antibody- or small molecule inhibitor-based therapies to target ovarian cancer as a model for ongoing and future integration of clinical therapeutic research. There are limited treatment options currently available. Although some targeted therapy agents show superior efficacy, the clinical outcomes of a majority of monotherapies have been disappointing (42). In practice, however, the clinical trial results have shown promise in patients who respond. Targeted therapies may be effective 


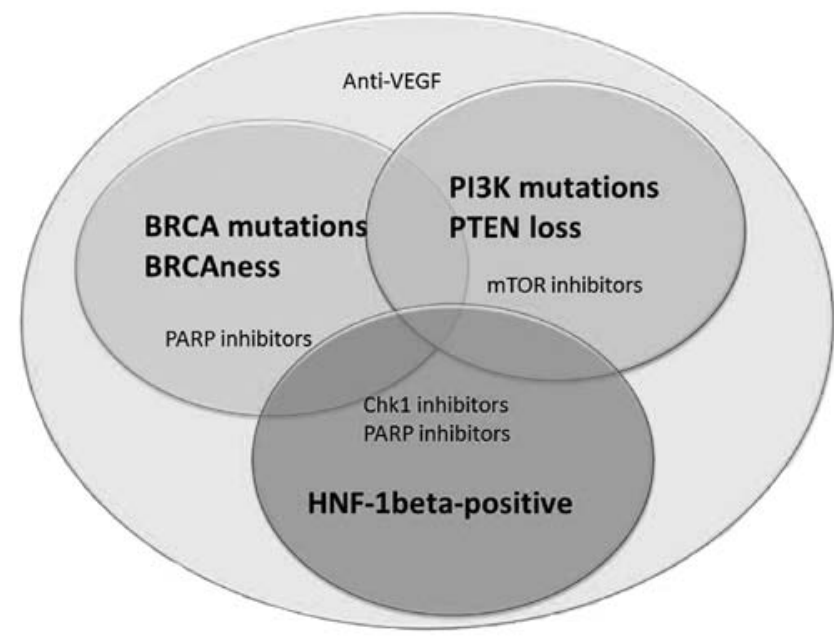

Figure 2. Venn diagram of ovarian cancer subtypes. Selected targeted treatment strategies are depicted.

in a subset of patients. This is due to the high complexity of the signaling network of target molecules in each patient. Currently, targeted agents should be used in combination with conventional cytotoxic chemotherapy or in the adjuvant setting. Data are still inconclusive and definitive conclusions on efficacy cannot yet be drawn.

Among many targeted agents, a promising, novel class of drugs for special patient populations of epithelial ovarian cancer expected to improve the effectiveness of current therapy include inhibitors of angiogenesis, PARP and DNA repair mechanisms (Fig. 2). Response rate of bevacizumab monotherapy in phase II trials was achieved in $20 \%$ of patients. Two randomized phase III trials (GOG-218 and ICON7) showed a PFS benefit of combination chemotherapy (paclitaxel + carboplatin) plus bevacizumab with maintenance bevacizumab. Despite a transient response, bevacizumab might be the most promising drug.

PARP inhibitors are promising candidates for targeted therapy. These inhibitors may work only in certain tumor subsets. A clinical trial assessed the efficacy and safety of a PARP inhibitor, olaparib, in patients with advanced BRCAmutated breast cancer (68). The objective response rate was $41 \%$ of patients. This result shows that olaparib is effective in patients with tumors that have loss of function of BRCA1/2associated DNA repair, namely, BRCA germline mutations or BRCA deficient-like (BRCAness) ovarian cancers. BRCA immunohistochemical negativity without BRCA mutations also shows a dysfunction of the HR system. In general, impaired ability to repair DNA by HR increases their chemosensitivity, suggesting that ovarian cancer cells arising in a background of BRCA dysfunction are more sensitive than other cells to DNA-damaging agents. Among sporadic ovarian cancer patients, a subset with BRCA dysfunction may be predictive of sensitivity to platinum-based chemotherapy, even if they do not harbor a BRCA1/2 germline mutation (53). PARP inhibitors appear to induce synthetic lethality in BRCAdeficient cells. Thus, these agents chemosensitize cancer cells with DNA double strand break repair defects.

Clear cell carcinoma is relatively less responsive to conventional carboplatin and paclitaxel-based chemotherapy which is associated with worse prognosis than serous and endometrioid histotypes. The mechanisms underlying CCC's poor response to standard chemotherapy regimens have not been understood. A recent report showed that reduced CDK2 (cyclin-dependent kinase 2) activity contributes to resistance to chemotherapy, because reduced cell proliferation is associated with chemoresistance (69). Furthermore, the genome-wide transcriptional analysis of CCC cell lines and tissue samples contribute to our understanding of the nature of CCC at the cell level. These analyses revealed that HNF-1 $\beta$ expression is specifically upregulated in CCC and correlates with cell survival and anti-apoptosis. The HNF-1 $\beta$-dependent pathway provides new insight into regulation of resistance to anticancer agents; checkpoint kinases are persistently activated in response to HNF-1 $\beta$, thus representing a barrier which limits therapeutic efficacy; and HNF-1 $\beta$-overexpressing cells are selectively sensitive to Chk1 inhibitors. These facts may provide support for the synthetic lethality concept. Cell cycle checkpoints provide a key mechanism for the innate cellular defense that occurs in response to environmental stresses including chemotherapeutic agents or oxidative stress (63).

Exploration of the highly potent targets within the network involved in HR repair has led to the discovery of Chk1 inhibitors, indicating that HR repair inhibition contributes to sensitization by Chk1 inhibition (104). Chk1 inhibition selectively sensitizes p53 mutant cells or possibly BRCAness ovarian cancer cells (70). The BRCAness profile harbors not only defects in HR or other DNA repair pathways, but also persistent activation of Chk1 activity due to the upregulation of HNF-1 $\beta$ expression. Furthermore, ATM and ATR, upstream targets of checkpoint kinases, also remain a critical target for cancer treatment because activated ATM signaling prolongs G2/M checkpoint arrest. Thus, inhibition of PARP1 (via olaparib), HR repair (mediated by Chk1 via AZD7762) or ATM (via KU-60019) would selectively sensitize p53 mutation, BRCAness phenotype (serous type ovarian cancer) or HNF-19\%-overexpressing tumor cells (clear cell type ovarian cancer) to chemotherapeutic agents.

Finally, clinical evaluation of molecularly targeted agents may not conform to traditional clinical trial designs. Therapeutic response is likely to be limited to a small subpopulation of patients possibly through germline mutations, somatic mutational or functional heterogeneity between ovarian cancers with the same histopathological subtype. The disappointing results may arise due to insufficient study power stemming from patient heterogeneity. There has been a paradigm shift in molecular targeted therapy away from targeting individual molecules to whole biological pathways. We must individualize the treatment option of ovarian cancer based on individualized molecular profiling in the context of personalized medicine. In addition, we currently need the potential predictive biomarkers to select the optimal type of chemotherapy and also the patients with the best chances of benefiting from targeted therapy.

In conclusion, it appears that inhibitors of angiogenesis, PARP and DNA repair mechanisms may hold promise as an adjunct drug in ovarian cancer therapy. The parallel or cross-talk pathway targeting strategy might be especially important in patients with coexisting angiogenesis pathway alterations and DNA repair deficiency. We must identify a 
very highly selected population most likely to show a real benefit in survival from molecular targeted drugs. Further prospective biomarker-led clinical trials are warranted for patient selection.

\section{Acknowledgements}

This study was supported by a Grant-in-aid for Scientific Research to H.K. from the Ministry of Education, Science and Culture of Japan.

\section{References}

1. Gaitskell K, Martinek I, Bryant A, Kehoe S, Nicum S and Morrison J: Angiogenesis inhibitors for the treatment of ovarian cancer. Cochrane Database Syst Rev 9: CD007930, 2011.

2. Banerjee $S$ and Kaye S: The role of targeted therapy in ovarian cancer. Eur J Cancer 47 (Suppl 3): S116-S130, 2011.

3. Oei AL, Sweep FC, Thomas CM, Boerman OC and Massuger LF: The use of monoclonal antibodies for the treatment of epithelial ovarian cancer (Review). Int J Oncol 32: 1145-1157, 2008.

4. Reichert JM: Antibody-based therapeutics to watch in 2011. MAbs 3: 76-99, 2011.

5. Itamochi H: Targeted therapies in epithelial ovarian cancer: molecular mechanisms of action. World J Biol Chem 1: 209-220, 2010.

6. Richardson DL, Backes FJ, Seamon LG, et al: Combination gemcitabine, platinum, and bevacizumab for the treatment of recurrent ovarian cancer. Gynecol Oncol 111: 461-466, 2008

7. Jurado JM, Sánchez A, Pajares B, Pérez E, Alonso L and Alba E: Combined oral cyclophosphamide and bevacizumab in heavily pre-treated ovarian cancer. Clin Transl Oncol 10: 583-586, 2008

8. Perren TJ, Swart AM, Pfisterer J, et al: A phase 3 trial of bevacizumab in ovarian cancer. N Engl J Med 365: 2484-2496, 2011.

9. Köhne $\mathrm{CH}$ : How to integrate molecular targeted agents in the continuum of care. Ann Oncol 21 (Suppl 7): vii134-vii139, 2010.

10. Moroney JW, Sood AK and Coleman RL: Aflibercept in epithelial ovarian carcinoma. Future Oncol 5: 591-600, 2009.

11. Teng LS, Jin KT, He KF, Zhang J, Wang HH and Cao J: Clinical applications of VEGF-trap (aflibercept) in cancer treatment. J Chin Med Assoc 73: 449-456, 2010.

12. Colombo N, Mangili G, Mammoliti S, et al: A phase II study of aflibercept in patients with advanced epithelial ovarian cancer and symptomatic malignant ascites. Gynecol Oncol 125: 42-47, 2012.

13. Coleman RL, Duska LR, Ramirez PT, et al: Phase 1-2 study of docetaxel plus aflibercept in patients with recurrent ovarian, primary peritoneal, or fallopian tube cancer. Lancet Oncol 12: 1109-1117, 2011.

14. Neal $\mathbf{J}$ and Wakelee H: AMG-386, a selective angiopoietin1/-2-neutralizing peptibody for the potential treatment of cancer. Curr Opin Mol Ther 12: 487-495, 2010.

15. Karlan BY, Oza AM, Richardson GE, et al: Randomized, doubleblind, placebo-controlled phase II Study of AMG 386 combined with weekly paclitaxel in patients with recurrent ovarian cancer. J Clin Oncol 30: 362-371, 2012.

16. Yarom $\mathrm{N}$ and Jonker DJ: The role of the epidermal growth factor receptor in the mechanism and treatment of colorectal cancer. Discov Med 11: 95-105, 2011.

17. Steffensen KD, Waldstrøm M, Jeppesen U, Jakobsen E, Brandslund I and Jakobsen A: The prognostic importance of cyclooxygenase 2 and HER2 expression in epithelial ovarian cancer. Int J Gynecol Cancer 17: 798-807, 2007.

18. Farley J, Fuchiuji S, Darcy KM, et al: Associations between ERBB2 amplification and progression-free survival and overal survival in advanced stage, suboptimally-resected epithelial ovarian cancers: a Gynecologic Oncology Group Study. Gynecol Oncol 113: 341-347, 2009

19. Tang $\mathrm{L}$ and Zhao X: Polyclonal antitumor immunoglobulin may play a role in ovarian cancer adjuvant therapy. Med Hypotheses 76: $530-532,2011$

20. Makhija S, Amler LC, Glenn D, et al: Clinical activity of gemcitabine plus pertuzumab in platinum-resistant ovarian cancer, fallopian tube cancer, or primary peritoneal cancer. J Clin Oncol 28: 1215-1223, 2010.
21. Langdon SP, Faratian D, Nagumo Y, Mullen P and Harrison DJ: Pertuzumab for the treatment of ovarian cancer. Expert Opin Biol Ther 10: 1113-1120, 2010.

22. Sheng Q, Liu X, Fleming E, et al: An activated ErbB3/NRG1 autocrine loop supports in vivo proliferation in ovarian cancer cells. Cancer Cell 17: 298-310, 2010.

23. Konner JA, Bell-McGuinn KM, Sabbatini P, et al: Farletuzumab, a humanized monoclonal antibody against folate receptor alpha, in epithelial ovarian cancer: a phase I study. Clin Cancer Res 16: 5288-5295, 2010.

24. Berek J, Taylor P, McGuire W, Smith LM, Schultes B and Nicodemus CF: Oregovomab maintenance monoimmunotherapy does not improve outcomes in advanced ovarian cancer. J Clin Oncol 27: 418-425, 2009.

25. Sabbatini P, Dupont J, Aghajanian C, et al: Phase I study of abagovomab in patients with epithelial ovarian, fallopian tube, or primary peritoneal cancer. Clin Cancer Res 12: 5503-5510, 2006.

26. Grisham RN, Berek J, Pfisterer J and Sabbatini P: Abagovomab: an anti-idiotypic CA-125 targeted immunotherapeutic agent for ovarian cancer. Immunotherapy 3: 153-162, 2011.

27. Ruf $\mathrm{P}$ and Lindhofer $\mathrm{H}$ : Induction of a long-lasting antitumor immunity by a trifunctional bispecific antibody. Blood 98: 2526-2534, 2001.

28. Baumann K, Pfisterer J, Wimberger P, et al: Intraperitoneal treatment with the trifunctional bispecific antibody Catumaxomab in patients with platinum-resistant epithelial ovarian cancer: a phase IIa study of the AGO Study Group. Gynecol Oncol 123: 27-32, 2011.

29. Bell-McGuinn KM, Matthews CM, Ho SN, et al: A phase II, single-arm study of the anti- $\alpha 5 \beta 1$ integrin antibody volociximab as monotherapy in patients with platinum-resistant advanced epithelial ovarian or primary peritoneal cancer. Gynecol Oncol 121: 273-279, 2011.

30. Matei D, Sill MW, Lankes HA, et al: Activity of sorafenib in recurrent ovarian cancer and primary peritoneal carcinomatosis: a gynecologic oncology group trial. J Clin Oncol 29: 69-75, 2011.

31. Bodnar L, Górnas M and Szczylik C: Sorafenib as a third line therapy in patients with epithelial ovarian cancer or primary peritoneal cancer: a phase II study. Gynecol Oncol 123: 33-36, 2011.

32. Ramasubbaiah R, Perkins SM, Schilder J, et al: Sorafenib in combination with weekly topotecan in recurrent ovarian cancer, a phase I/II study of the Hoosier Oncology Group. Gynecol Oncol 123: 499-504, 2011.

33. Welch SA, Hirte HW, Elit L, et al: Sorafenib in combination with gemcitabine in recurrent epithelial ovarian cancer: a study of the Princess Margaret Hospital Phase II Consortium. Int J Gynecol Cancer 20: 787-793, 2010.

34. Pölcher M, Eckhardt M, Coch C, et al: Sorafenib in combination with carboplatin and paclitaxel as neoadjuvant chemotherapy in patients with advanced ovarian cancer. Cancer Chemother Pharmacol 66: 203-207, 2010.

35. Eichbaum M, Mayer C, Eickhoff R, et al: The PACOVAR-trial: a phase I/II study of pazopanib (GW786034) and cyclophosphamide in patients with platinum-resistant recurrent, pre-treated ovarian cancer. BMC Cancer 11: 453, 2011.

36. Friedlander M, Hancock KC, Rischin D, et al: A Phase II, open-label study evaluating pazopanib in patients with recurrent ovarian cancer. Gynecol Oncol 119: 32-37, 2010.

37. Patel BB, He YA, Li XM, et al: Molecular mechanisms of action of imatinib mesylate in human ovarian cancer: a proteomic analysis. Cancer Genomics Proteomics 5: 137-149, 2008.

38. Santen RJ, Brodie H, Simpson ER, Siiteri PK and Brodie A: History of aromatase: saga of an important biological mediator and therapeutic target. Endocr Rev 30: 343-375, 2009.

39. Juretzka M, Hensley ML, Tew W, et al: A phase 2 trial of oral imatinib in patients with epithelial ovarian, fallopian tube, or peritoneal carcinoma in second or greater remission. Eur J Gynaecol Oncol 29: 568-572, 2008.

40. Blank SV, Christos P, Curtin JP, et al: Erlotinib added to carboplatin and paclitaxel as first-line treatment of ovarian cancer: a phase II study based on surgical reassessment. Gynecol Oncol 119: 451-456, 2010.

41. Nimeiri HS, Oza AM, Morgan RJ, et al: Efficacy and safety of bevacizumab plus erlotinib for patients with recurrent ovarian, primary peritoneal, and fallopian tube cancer: a trial of the Chicago, PMH, and California Phase II Consortia. Gynecol Oncol 110: 49-55, 2008. 
42. Garcia AA, Sill MW, Lankes HA, et al: A phase II evaluation of lapatinib in the treatment of persistent or recurrent epithelial ovarian or primary peritoneal carcinoma: A gynecologic oncology group study. Gynecol Oncol 124: 569-574, 2012.

43. Kimball KJ, Numnum TM, Kirby TO, et al: A phase I study of lapatinib in combination with carboplatin in women with platinum sensitive recurrent ovarian carcinoma. Gynecol Onco 111: 95-101, 2008

44. Weroha SJ, Oberg AL, Ziegler KL, et al: Phase II trial of lapatinib and topotecan (LapTop) in patients with platinum-refractory/ resistant ovarian and primary peritoneal carcinoma. Gynecol Oncol 122: 116-120, 2011

45. Murphy M and Stordal B: Erlotinib or gefitinib for the treatment of relapsed platinum pretreated non-small cell lung cancer and ovarian cancer: a systematic review. Drug Resist Updat 14 177-190, 2011.

46. Gnoni A, Marech I, Silvestris N, Vacca A and Lorusso V: Dasatinib: an anti-tumour agent via Src inhibition. Curr Drug Targets 12: 563-578, 2011.

47. Le XF, Mao W, Lu Z, Carter BZ and Bast RC Jr: Dasatinib induces autophagic cell death in human ovarian cancer. Cancer 116: 4980-4990, 2010

48. Oza AM, Elit L, Tsao MS, et al: Phase II study of temsirolimus in women with recurrent or metastatic endometrial cancer: a tria of the NCIC Clinical Trials Group. J Clin Oncol 29: 3278-3285, 2011.

49. Behbakht K, Sill MW, Darcy KM, et al: Phase II trial of the mTOR inhibitor, temsirolimus and evaluation of circulating tumor cells and tumor biomarkers in persistent and recurrent epithelial ovarian and primary peritoneal malignancies: a Gynecologic Oncology Group study. Gynecol Oncol 123: 19-26, 2011.

50. Temkin SM, Yamada SD and Fleming GF: A phase I study of weekly temsirolimus and topotecan in the treatment of advanced and/or recurrent gynecologic malignancies. Gynecol Oncol 117: 473-476, 2010

51. Kollmannsberger C, Hirte H, Siu LL, et al: Temsirolimus in combination with carboplatin and paclitaxel in patients with advanced solid tumors: a NCIC-CTG, phase I, open-label doseescalation study (IND 179). Ann Oncol 23: 238-244, 2012.

52. Janku F, Wheler JJ, Westin SN, et al: PI3K/AKT/mTOR inhibitors in patients with breast and gynecologic malignancies harboring PIK3CA mutations. J Clin Oncol 30: 777-782, 2012.

53. Radosa MP, Häfner N, Camara O, et al: Loss of BRCA1 protein expression as indicator of the BRCAness phenotype is associated with favorable overall survival after complete resection of sporadic ovarian cancer. Int J Gynecol Cancer 21: 1399-1406, 2011.

54. Narod SA: BRCA mutations in the management of breast cancer: the state of the art. Nat Rev Clin Oncol 7: 702-707, 2010.

55. Javle M and Curtin NJ: The potential for poly (ADP-ribose) polymerase inhibitors in cancer therapy. Ther Adv Med Oncol 3: 257-267, 2011.

56. Lord CJ and Ashworth A: Targeted therapy for cancer using PARP inhibitors. Curr Opin Pharmacol 8: 363-369, 2008.
57. Turner N, Tutt A and Ashworth A: Hallmarks of 'BRCAness' in sporadic cancers. Nat Rev Cancer 4: 814-819, 2004.

58. Penning TD: Small-molecule PARP modulators - current status and future therapeutic potential. Curr Opin Drug Discov Devel 13: 577-586, 2010

59. Fong PC, Yap TA, Boss DS, et al: Poly(ADP)-ribose polymerase inhibition: frequent durable responses in BRCA carrier ovarian cancer correlating with platinum-free interval. J Clin Oncol 28 : 2512-2519, 2010

60. Gelmon KA, Tischkowitz M, Mackay H, et al: Olaparib in patients with recurrent high-grade serous or poorly differentiated ovarian carcinoma or triple-negative breast cancer: a phase 2 , multicentre, open-label, non-randomised study. Lancet Oncol 12: 852-861, 2011

61. Welch S, Hirte HW, Carey MS, et al: UCN-01 in combination with topotecan in patients with advanced recurrent ovarian cancer: a study of the Princess Margaret Hospital Phase II consortium. Gynecol Oncol 106: 305-310, 2007.

62. Morgan MA, Parsels LA, Zhao LP, et al: Mechanism of radiosensitization by the Chk1/2 inhibitor AZD7762 involves abrogation of the G2 checkpoint and inhibition of homologous recombinational DNA repair. Cancer Res 70: 4972-4981,2010.

63. Kajihara H, Yamada Y, Kanayama S, et al: Clear cell carcinoma of the ovary: potential pathogenic mechanisms (Review). Oncol Rep 23: 1193-1203, 2010.

64. Yamada Y, Shigetomi H, Onogi A, et al: Redox-active ironinduced oxidative stress in the pathogenesis of clear cell carcinoma of the ovary. Int J Gynecol Cancer 21: 1200-1207, 2011.

65. Smyth JF, Gourley C, Walker G, et al: Antiestrogen therapy is active in selected ovarian cancer cases: the use of letrozole in estrogen receptor-positive patients. Clin Cancer Res 13: 3617-3622, 2007.

66. Fang F, Balch C, Schilder J, et al: A phase 1 and pharmacodynamic study of decitabine in combination with carboplatin in patients with recurrent, platinum-resistant, epithelial ovarian cancer. Cancer 116: 4043-4053, 2010.

67. Modesitt SC, Sill M, Hoffman JS, Bender DP and Gynecologic Oncology Group: A phase II study of vorinostat in the treatment of persistent or recurrent epithelial ovarian or primary peritoneal carcinoma: a Gynecologic Oncology Group study. Gynecol Oncol 109: 182-186, 2008.

68. Tutt A, Robson M, Garber JE, et al: Oral poly(ADP-ribose) polymerase inhibitor olaparib in patients with BRCA1 or BRCA2 mutations and advanced breast cancer: a proof-of-concept trial. Lancet 376: 235-244, 2010.

69. Itamochi H, Yoshida T, Walker CL, et al: Novel mechanism of reduced proliferation in ovarian clear cell carcinoma cells: cytoplasmic sequestration of CDK2 by p27. Gynecol Oncol 122: 641-647, 2011.

70. Vance S, Liu E, Zhao L, et al: Selective radiosensitization of p53 mutant pancreatic cancer cells by combined inhibition of Chk1 and PARP1. Cell Cycle 10: 4321-4329, 2011. 